\title{
Effect of alpha 2,6 Sialylation on Integrin-mediated Adhesion of Breast Cancer Cells to Fibronectin and Collagen IV
}

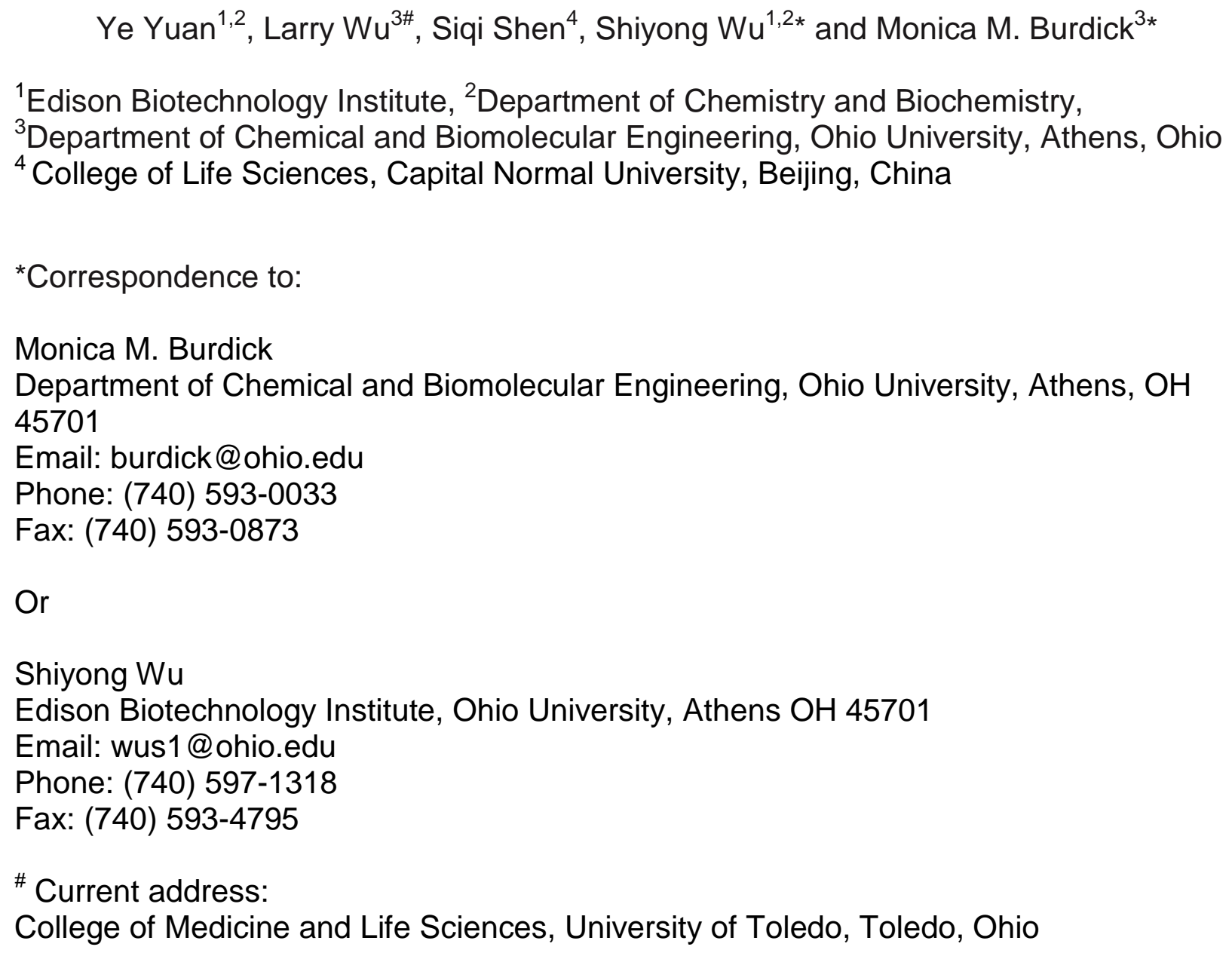


2 Aims: To determine the role of sialylation on $\alpha 5 \beta 1$ and $\alpha 2 \beta 1$ integrins in the regulation

3 of adhesion between breast cancer cells and extracellular matrix (ECM).

4 Main methods: Static cell adhesion assays were performed to quantify avidity of breast

5 cancer cells to ECM. The effects of sialidases on $\alpha 2,6$ sialylation was assessed by flow cytometry using biotin conjugated sambucus nigra lectin. Lectin affinity assays were

7 used to determine expression of $\alpha 2,6$ sialylated integrins. Cell migration and invasion were investigated by wound healing and transwell invasion assays.

9 Key findings: $\alpha 2, \alpha 5$ and $\beta 1$ integrins had considerable $\alpha 2,6$ sialylation on MDA-MB-231 10 cells, whereas signals from MCF-7 cells were undetectable. Cleavage of $\alpha 2,6$ sialylation 11 increased adhesion of MDA-MB-231 cells to ECM, while adhesion of MCF-7 cells was 12 unaffected, consistent with the latter's lack of endogenous $\alpha 2,6$ sialylated surface 13 integrins. Neither surface expression of $\alpha 2 \beta 1$ and $\alpha 5 \beta 1$ integrins, nor activated $\beta 1$ 14 integrin, changed in MDA-MB-231 cells after sialidase treatment. However, sialidase 15 treatment did not have significant impact on migration or invasion of MDA-MB-231 cells.

16 Significance: Cell adhesion is an important early step of cancer metastasis, yet the roles 17 of sialylation in regulating integrin-mediated breast cancer cell adhesion in comparison 18 to migration and invasion are not well-understood. Our data suggest desialylation of $19 \alpha 2,6$-sialylated integrins increases adhesion, but not migration or invasion, of MDA-MB-

20231 cells to ECM without altering integrin expression. It should be considered that $\alpha 2,6$ 21 sialylation may play different roles in regulating cell adhesion of different cancer cells 22 when developing potential therapeutics targeting $\alpha 2,6$ sialylation. 
2 Abbreviated title: $\alpha 2,6$ sialylation Regulates Integrins Avidity

3

4 Key words: cell adhesion; extracellular matrix; integrins; N-Acetylneuraminic acid;

5 breast neoplasms

6 
The avidity of integrins to their ligands is not only affected by surface expression

4 level, but also regulated by glycosylation. Integrins, glycoproteins composed of $\alpha$ and $\beta$

5 subunits, are known receptors of extracellular matrix (ECM) (Hynes, 2002). The

6 interactions between integrins and ECM have been shown to mediate diverse

7 physiological functions such as cell adhesion, development and carcinogenesis

8 (Bianchi-Smiraglia et al. , 2012). As a basic function carried out by integrins, adhesion

9 to ECM on endothelial and stromal cells is thought to participate in cancer cell

10 metastasis by facilitating extravasation of circulating cancer cells (Lafrenie et al. , 1992).

11 In addition, integrin mediated adhesion also contributes to the resistance of cancer cells

12 to chemotherapeutic drugs (Aoudjit and Vuori, 2012) and ionizing radiation (Eke et al. ,

13 2012). The function of integrins is not only regulated by surface protein expression, but

14 is also controlled by signaling pathways, which confer conformation changes between

15 low and high affinity states (Arnaout et al. , 2007, Askari et al. , 2009).

16 The alteration of integrin sialylation is also an important regulator of cell

17 adhesion, especially sialylation of $\beta 1$ integrin. Mitogen-activated protein kinase/ERK

18 kinase (MEK) activation in U937 myeloid cells induced hyposialylation of $\beta 1$ integrin and 19 increased the avidity of U937 cell adhesion to fibronectin (Seales et al. , 2005b). In

20 contrast, upregulation of ST6Gal-I in metastatic colon cancer cells led to the elevation of $21 \alpha 2,6$ sialylated $\beta 1$ integrin, yet also enhanced cell adhesion, migration and metastasis

22 (Seales et al. , 2005a, Shaikh et al. , 2008). Ras activation increased $\alpha 2,6$ sialylation of $23 \quad \beta 1$ integrin of HD3 colonocytes, and desialylation by sialidase inhibited cell adhesion to 
1 collagen I (Seales et al. , 2003). Application of a fluorinated sialic acid analogue has

2 shown great potential in reducing B16F10 melanoma cell adhesion to ECM and tumor

3 growth in mice, which is encouraging for developing drugs targeting hypersialylation in

4 cancer (Bull et al. , 2013). In light of the importance of $\alpha 2,6$ sialylation of integrins in the

5 regulation of adhesion of these types of cells, we sought to determine the role of $\alpha 2,6$

6 sialylation of integrins in regulating adhesion and invasion of breast cancer cells to

7 ECM. Our data indicate that integrins are highly $\alpha 2,6$ sialylated on MDA-MB-231 cells

8 but not on MCF-7 cells. Desialylation of integrins increases adhesion of MDA-MB-231

9 cells to ECM without alteration of integrin expression. Moreover, the desialylation10 increased adhesion does not correlate with the migration and invasiveness of the cells.

11

\section{Materials and methods}

Cell Culture

The breast cancer cell line MDA-MB-231 was kindly provided by Dr. Jianjian Li (University of California Davis Cancer Center, CA), and the breast cancer cell line MCF7 was obtained from the American Type Culture Collection (Manassas, VA). MDA-MB231 cells were cultured in minimal essential medium (MEM) (Corning, Manassas, VA), containing $10 \%$ fetal bovine serum (Atlanta Biologicals, Lawrenceville, GA), $1 \mathrm{mM}$ sodium pyruvate, $1 \mathrm{X}$ non-essential amino acids (NEAA) and 1\% penicillin and streptomycin. MCF-7 cells were cultured in Dulbecco's Modified Eagle Medium (DMEM) supplemented with $10 \%$ fetal bovine serum and $1 \%$ penicillin and streptomycin. 
1

Antibodies and Reagents

Sialidase from Vibrio cholerae (Ada et al. , 1961) (VC, broad substrate specificity) was purchased from Roche (Indianapolis, IN). Clostridium perfringens sialidase (Cassidy et al. , 1965, St Geme, 1994) (CP, specificity of cleavage: $\alpha 2,3>\alpha 2,6$ sialylation) and Arthrobacter ureafaciens sialidase (Corfield et al. , 1983, Saito et al. , 1979) (AU, specificity of cleavage: $\alpha 2,6>\alpha 2,3$ sialylation) were purchased from SigmaAldrich (St. Louis, MO) and Prozyme (Hayward, CA), respectively. An ECM screening kit containing fibronectin and collagen IV pre-coated strips was obtained from EMD Millipore (Billerica, MA). The following anti-human primary antibodies were purchased from BD Biosciences (San Jose, CA): CD29 (Mab13) mAb, CD15 (HI98) mAb, CD15s (CSLEX1) mAb, and CD49b (12F1) mAb. The following anti-human primary antibodies were obtained from Santa Cruz Biotechnology (Santa Cruz, CA): CD49e (JBS5) mAb, CD49e (H-104) polyclonal antibody, CD49b (H-293) polyclonal antibody and CD29 (N20) polyclonal antibody. Anti-activated integrin $\beta 1$ antibody (HUTS-4) was purchased from EMD Millipore (Billerica, MA). Biotin conjugated Sambucus nigra lectin (SNA) was obtained from EY labs (San Mateo, CA). Corresponding isotype control antibodies, fluorescein isothiocyanate (FITC) conjugated secondary antibodies, and FITCconjugated streptavidin were purchased from BD Biosciences. Horse radish peroxidase (HRP) conjugated secondary antibodies were obtained from Santa Cruz Biotechnology. Streptavidin agarose resin was obtained from Thermo Scientific (Rockford, IL). 
Western Blotting and Lectin Affinity Assay

For western blotting, MDA-MB-231 and MCF-7 cells were first lysed in 2\% NP-40 buffer containing protease inhibitor on ice. Supernatant was collected by centrifugation. Proteins $(100 \mu \mathrm{g})$ were separated in $8 \%$ sodium dodecyl sulfate polyacrylamide gel electrophoresis (SDS-PAGE) resolving gel and transferred to a nitrocellulose membrane (Pall, Port Washington, NY). After blocking for $1 \mathrm{~h}$ with 5\% non-fat milk in Tris-buffered saline with Tween (TBS-T) at room temperature, the membrane was incubated with anti-CD49b (H293), anti-CD49e (H-104), or anti-CD29 (N-20) antibodies at $4^{\circ} \mathrm{C}$ overnight. After washing with TBS-T three times, the membrane was incubated with corresponding HRP conjugated secondary antibodies for $30 \mathrm{~min}$ at room temperature. Blots were washed with TBS-T and Tris-buffered saline (TBS) twice respectively and visualized using West Pico Supersignal chemiluminescent substrate (Pierce, Rockford, IL). $\beta$-actin was used as the loading control.

For the lectin affinity assay, cell lysates $(300 \mu \mathrm{g})$ of MDA-MB-231 and MCF-7 cells were incubated with $50 \mu \mathrm{g} / \mathrm{ml}$ biotinylated SNA, a specific lectin for $\alpha 2,6$ sialylation (Shibuya et al. , 1987, Taatjes et al. , 1988), at $4^{\circ} \mathrm{C}$ overnight. Streptavidin agarose resin (Thermo Scientific, Waltham, MA) was added to cell lysates for $4 \mathrm{~h}$ rotation at $4^{\circ} \mathrm{C}$. The beads were then washed once with TBS/1\% Triton X-100 and twice with TBS/0.5\% Triton X-100. Bead-protein complexes were boiled in SDS-PAGE loading buffer, and released proteins were electrophoresed on an 8\% SDS-PAGE as described above. Since $\beta$-actin cannot be precipitated by the resin, the loading control ( $\beta$-actin) for lectin affinity assay was run in a separate gel using the cell lysates before adding biotinylated SNA. 
Surface sialylation and integrin expression were assessed by flow cytometry. MDA-MB-231 and MCF-7 cells were collected in phosphate buffered saline (PBS) and washed twice. Cells were then fixed in $4 \%$ formaldehyde for $10 \mathrm{~min}$. After being chilled on ice for $60 \mathrm{~s}$, cells were washed once in PBS, and twice in $0.5 \%$ bovine serum

6 albumin (BSA) before resuspension in $0.5 \%$ BSA for blocking. $1 \times 10^{6}$ cells were 7 incubated with primary mAb anti-CD15s (CSLEX1), anti-CD15 (HI98), anti-CD49b 8 (12F1), anti-CD49e (JBS5), anti-CD29 (Mab13), anti-CD29 (HUTS-4), biotinylated SNA 9 and corresponding isotype control antibodies for $1 \mathrm{~h}$ at room temperature. For biotinylated SNA, the FITC-conjugated streptavidin was used as background control.

11 For CD15 and CD15s, the secondary antibody alone was used as background. After 12 washing with $0.5 \%$ BSA twice, cells were incubated with corresponding FITC 13 conjugated secondary antibodies for $30 \mathrm{~min}$ at room temperature. Cells were washed twice with $0.5 \%$ BSA, resuspended in $0.5 \mathrm{ml} \mathrm{PBS}$ and analyzed on a FACSAria Special Order Research Product flow cytometer (BD Biosciences).

Cell Adhesion Assay

Adhesion of MDA-MB-231, MCF-7 and HT-29 cells to fibronectin was measured using ECM coated strips (EMD Millipore) per the manufacturer's instructions. Briefly, cells were collected, washed and resuspended in culture medium. $2 \times 10^{5}$ MDA-MB-231

21 cells, $3 \times 10^{5}$ MCF-7 cells and $1 \times 10^{5}$ HT-29 cells were seeded onto fibronectin coated

22 strips. Cells were incubated for $30 \mathrm{~min}$ at $37^{\circ} \mathrm{C}$. Strips were washed by DPBS to remove 23 non-adherent cells. $0.2 \%$ Crystal violet in $10 \%$ ethanol was used to stain attached cells 
1 and the cells were finally dissolved in 2\% SDS after washing. Absorbance at $540 \mathrm{~nm}$ was measured by a microplate reader (Molecular Devices, Sunnyvale, CA). Absorbance was normalized to the control cells incubated in DPBS without sialidase.

4

Cell Migration and Invasion Assay

For migration, a wound-healing assay was performed as previously described with modifications (Cheng et al. , 2012). A 96-well plate for suspension culture (Greiner

8 Bio-One, Monroe, NC) was coated with $2 \mu \mathrm{g} / \mathrm{cm}^{2}$ fibronectin overnight at $4^{\circ} \mathrm{C}$. Then the cells were mock treated or treated with sialidases, and monolayer wounds were created using a $200 \mu \mathrm{L}$ pipette tip. The cells were washed once with serum free medium and

11 allowed to migrate for $7 \mathrm{~h}$ in FBS free DMEM. The wound healing was visualized using 12 an Olympus IX70 inverted microscope (Olympus, Tokyo, Japan) at 4X magnification. 13 Photographs were taken by a Retiga 1300 CCD Camera (Qimaging, Burnaby, B.C., in 24-well plates (Corning, Tewksbury, MA) were used per the manufacturer's instructions. Inner chambers of transwell inserts were first coated with $20 \mu \mathrm{g} / \mathrm{ml}$ fibronectin overnight at $4^{\circ} \mathrm{C}$. Cells were mock treated or treated with sialidases. After washing once with serum free medium, the cells $\left(1 \times 10^{5}\right)$ were suspended in $100 \mu \mathrm{L}$

22 FBS free medium and seeded onto each inner chamber. $600 \mu \mathrm{L}$ DMEM with 10\% FBS 23 was added to the outer chambers. Transwell inserts were incubated for $24 \mathrm{~h}$ at $37^{\circ} \mathrm{C}$. 
1 Cells that migrated into the lower surface of the membrane were fixed by $4 \%$

2 formaldehyde and stained with crystal violet. In each experiment, cells were counted in

34 different fields. Cells were imaged by the same digital camera and software as

4 described above.

5

6 Statistical Analysis

The data are presented as mean \pm SD of at least 3 independent experiments. Student's $t$-test was used in comparing control group and each treatment. Only $p<0.05$ was considered statistically significant.

\section{Results}

$13 \alpha 5 \beta 1$ and $\alpha 2 \beta 1$ integrins on MDA-MB-231 but not MCF-7 cells are $\alpha 2,6$ sialylated

first determined the expression and sialylation on $\alpha 5 \beta 1$ and $\alpha 2 \beta 1$ integrin, which play

critical roles in regulation of adhesion of breast cancer MDA-MB-231 or MCF-7 cells to

17 ECM proteins, specifically collagen IV (Col IV) and fibronectin (Lee et al. , 2014). Our

18 data indicate that $\alpha 2, \alpha 5$ and $\beta 1$ integrins were expressed in both cell lines, and MDA-

19 MB-231 expressed a higher level of each integrin compared to MCF-7 cells (Fig 1, right

20 panel). In view of the importance of $\alpha 2,6$ sialylation in regulating cell adhesion, lectin

21 SNA was used as an $\alpha 2,6$ sialylation marker to compare the sialylation level between

22 the two breast cancer cell lines. However, only the integrins on MDA-MB-231 were

23 heavily $\alpha 2,6$ sialylated; the $\alpha 2,6$ sialylation of integrins on MCF-7 cells was nearly 
1 undetectable by lectin affinity assay (Fig 1, left panel).

3 Desialylation of $\alpha 2,6$-sialylated integrins increases adhesive affinity between MDA-MB-

4231 cells and ECM proteins

5 To determine the involvement of $\alpha 2,6$ sialylation in mediating breast cancer cell

6 adhesion, we tested the effect of three sialidases with different specificities on adhesive

7 affinities between $\alpha 2 \beta 1$ integrin and Col IV, and between $\alpha 5 \beta 1$ and fibronectin. $V$.

8 cholera (VC) sialidase has broad cleavage activity (i.e., no specificity preference for

9 sialic acid linkages) (Ada et al., 1961); and C. perfringens (CP) and A. ureafaciens (AU)

10 sialidases have a preference towards $\alpha 2,3$ sialylation (Cassidy et al., 1965, St Geme,

11 1994) and $\alpha 2,6$ sialylation (Corfield et al., 1983, Saito et al., 1979), respectively. Our

12 data showed that the attachment of MDA-MB-231 cells to collagen IV was increased to

$13142.2 \% \pm 19.9 \%$ or $123.1 \% \pm 15.4 \%$ after $\mathrm{CP}$ sialidase or AU sialidase treatment,

14 respectively (Fig 2A). The attachment of MDA-MB-231 cells to fibronectin was

15 increased to $131.3 \% \pm 10.7 \%$ and $138.1 \% \pm 19.7 \%$ after CP sialidase and AU sialidases

16 treatment, respectively (Fig 2A). As expected, under the same conditions, the

17 attachment of MCF-7 cells to collagen IV or fibronectin did not increase (Fig 2B).

18 However, it was a surprise that VC sialidase had no statistically significant effect on

19 breast cancer cell adhesion, given its ability to cleave sialic acids of multiple linkages

20 (Fig 2A). Further analysis using a sialylated integrins-expressing colon cancer cell line

21 HT-29 (Vercoutter-Edouart et al. , 2008) demonstrated that the same VC sialidase

22 treatment decreased adhesion to collagen IV by $70 \% \pm 1.9 \%$ (Fig $2 \mathrm{C}$ ), which is in

23 agreement with a previous report (Kemmner et al. , 1992). Interestingly CP and AU 
1 sialidases had no statistically significant effects on HT-29 cell adhesion (Fig 2C),

2 suggesting the specificity of sialidases might be cell line dependent.

Since VC sialidase, which should cleave both $\alpha 2,6$ and $\alpha 2,3$ linked sialic acids,

4 did not have a significant effect on the cell adhesion, we examined specificities of the

5 sialidases using lectin SNA as $\alpha 2,6$ sialylation marker and CD15s/CD15 ratio as $\alpha 2,3$

6 sialylation markers, in removing sialic acids. Our data showed that lectin SNA-binding

7 on MDA-MB-231 cells was reduced by $5.8 \% \pm 1.3 \%, 58.5 \% \pm 14.5 \%$ and $84.3 \% \pm 0.5 \%$

8 after treating with $\mathrm{VC}, \mathrm{CP}$ and $\mathrm{AU}$ sialidase respectively (Figs. $3 \mathrm{~A}, 3 \mathrm{C}$ ). This result

9 confirmed that $\mathrm{CP}$ and $\mathrm{AU}$ sialidases are more efficient in cleaving $\alpha 2,6$ sialylation than

10 VC sialidase. Our data also showed that endogenous CD15S expression on MDA-MB-

11231 cells was very weak and none of the sialidase treatments had any effect on

12 CD15S/CD15 ratio on the cells (Figs. 3A, 3C). As for MCF-7 cells, the lectin SNA-

13 binding and CD15S expression were hardly detected. Thus the sialidase treatment did

14 not have any effect on these markers as expected (Figs. 3B, 3D).

To eliminate the possibility that the changes in adhesion to Col IV and fibronectin

16 were due to the surface integrin expression level changes after sialidase treatment, flow

17 cytometry was used to analyze cells. In MDA-MB-231 cells, there was no significant

18 change in any integrins (Fig 4A). In MCF-7 cells, $\beta 1$ integrin level did not change after

19 sialidase treatment (Fig 4B). In addition, activated $\beta 1$ integrin was also tested under the

20 same conditions, and no significant change was observed in either cell line (Fig 4).

21 These data indicate that the increased adhesion resultant with sialidase treatments is

22 not due to a change in surface expression or activation of $\beta 1$ integrin. 
1 Sialidase-catalyzed integrin desialylation does not correlate with MDA-MB-231 cell

2 migration and invasion

To determine whether the desialylation and increased adhesion of MDA-MB-231

4 cells are correlated to the later metastatic cascade steps of migration and invasion, we

5 examined migration and invasion of desialylated cells on fibronectin-coated surface

6 using wound healing assay and transwell invasion assay. Our data indicate that CP or

7 AU sialidase treatments have no statistically significant effects on migration and

8 invasiveness of the cells (Fig 5). As CP and AU were confirmed to be the most efficient

9 sialidases cleaving $\alpha 2,6$ sialylation on MDA-MB-231 cells, these data together indicate

10 that decreasing $\alpha 2,6$ sialylation by sialidase treatment does not affect cell migration and

11 invasion, regardless of the influence on cell adhesion.

12

13

14

15

16

17

18

19

20

21

22

23

\section{Discussion}

Altered glycosylation of integrins plays roles in cancer cell adhesion, migration and invasion during metastasis (Almaraz et al. , 2012, Christie et al. , 2008, Lee et al. , 2010, Reddy and Kalraiya, 2006, Uemura et al. , 2009). As one of a diverse family of sialylated integrins, $\alpha 2,6$ sialylated $\beta 1$ integrin has been identified in many types of cancer cells, such as ovarian cancer cells (SKOV3 and PA1) (Christie et al., 2008), colon cancer cells (SW480, Lovo) (Lee et al., 2010), and melanoma cells (WM9 and WM239) (Kremser et al. , 2008). However, the role of sialylation on breast cancer cells in regulation of cell adhesion is not fully understood. It has been reported that antisense transfection of ST6 Gal-I decreased adhesion of MDA-MB-435 breast cancer cells to collagen IV (Lin et al. , 2002). But MDA-MB-435 cells were later found to originate from 
1 M14 melanoma cells (Rae et al. , 2007), leaving a need to investigate the differential

2 sialylation of integrins on widely used breast cancer cells, for instance MDA-MB-231

3 and MCF-7 cells, and the role(s) in cell adhesion. In this study, we determined the

4 regulatory roles of sialylation on $\alpha 5 \beta 1$ and $\alpha 2 \beta 1$ integrins, which mediate the adhesion

5 of breast cancer cells to ECM proteins (Lee et al., 2014). We demonstrated that MDA-

6 MB-231, but not MCF-7, cells are highly sialylated with $\alpha 2,6$ linked sialic acids on $\alpha 2, \alpha 5$

7 and $\beta 1$ subunits of the integrins (Figs. 1 and 3); and that $\alpha 2,6$ sialylation reduces the

8 affinity between MDA-MB-231 cells to collagen IV and fibronectin (Fig 2). These results

9 agree with previous findings with leukemic cells (Pretzlaff et al. , 2000, Seales et al., 2005b, Semel et al. , 2002). Nevertheless, the results contrast with large amount of

11 reports based on the studies of colon cancer cells indicating $\alpha 2,6$ sialylation on $\beta 1$ 12 integrin promotes the cell adhesion to ECM proteins laminin and collagens (Kemmner et 13 al., 1992, Seales et al., 2005a, Shaikh et al., 2008). Thus, our results further confirm 14 that the regulation of integrin function by sialylation is not uniform across different 15 cancer cell types. Moreover, our results also demonstrate that the sialylation-increased 16 adhesion of MDA-MB-231 cells is not correlated with migration and invasiveness of the 17 cells (Fig 5). These results, together with previous reports (Gu and Taniguchi, 2008, 18 Lawrence and Springer, 1991, Yamamoto et al. , 1997, Yamamoto et al. , 2001), 19 strongly indicate that sialylated integrin-mediated cancer adhesion does not have a 20 direct correlation with cancer cell migration and invasiveness. 
3 RO1CA086928 (to SW) and graduate assistantship (to Y. Yuan) from the Department of

4 Chemistry and Biochemistry, Ohio University. The authors thank Dr. Shinhee Lee,

5 Curran Rhodes, Tiantian Liu and Michelle Pate for their technical assistance, as well as

6 Grady Carlson for technical assistance and critical review of the manuscript. 
Ada GL, French EL, Lind PE. Purification and properties of neuraminidase from Vibrio cholerae. J Gen Microbiol. 1961;24:409-25.

Almaraz RT, Tian Y, Bhattarcharya R, Tan E, Chen SH, Dallas MR, et al. Metabolic flux increases glycoprotein sialylation: implications for cell adhesion and cancer metastasis. Mol Cell Proteomics. 2012;11:M112 017558.

Aoudjit F, Vuori K. Integrin signaling in cancer cell survival and chemoresistance. Chemother Res Pract. 2012;2012:283181.

Arnaout MA, Goodman SL, Xiong JP. Structure and mechanics of integrin-based cell adhesion. Curr Opin Cell Biol. 2007;19:495-507.

Askari JA, Buckley PA, Mould AP, Humphries MJ. Linking integrin conformation to function. J Cell Sci. 2009;122:165-70.

Bianchi-Smiraglia A, Paesante S, Bakin AV. Integrin beta5 contributes to the tumorigenic potential of breast cancer cells through the Src-FAK and MEK-ERK signaling pathways. Oncogene. 2012;32:3049-58.

Bull C, Boltje TJ, Wassink M, de Graaf AM, van Delft FL, den Brok MH, et al. Targeting aberrant sialylation in cancer cells using a fluorinated sialic acid analog impairs adhesion, migration, and in vivo tumor growth. Molecular cancer therapeutics. 2013;12:1935-46.

Cassidy JT, Jourdian GW, Roseman S. The sialic acids. VI. Purification and properties of sialidase from Clostridium perfringens. J Biol Chem. 1965;240:3501-6.

Cheng $\mathrm{H}$, Mollica MY, Lee SH, Wang L, Velazquez-Martinez CA, Wu S. Effects of nitric oxidereleasing nonsteroidal anti-inflammatory drugs (NONO-NSAIDs) on melanoma cell adhesion. Toxicology and applied pharmacology. 2012;264:161-6.

Christie DR, Shaikh FM, Lucas JAt, Lucas JA, 3rd, Bellis SL. ST6Gal-I expression in ovarian cancer cells promotes an invasive phenotype by altering integrin glycosylation and function. J Ovarian Res. 2008;1:3.

Corfield AP, Higa H, Paulson JC, Schauer R. The specificity of viral and bacterial sialidases for alpha(2-3)- and alpha(2-6)-linked sialic acids in glycoproteins. Biochim Biophys Acta. 1983;744:121-6.

Eke I, Dickreuter E, Cordes N. Enhanced radiosensitivity of head and neck squamous cell carcinoma cells by beta1 integrin inhibition. Radiother Oncol. 2012;104:235-42.

Gu J, Taniguchi N. Potential of N-glycan in cell adhesion and migration as either a positive or negative regulator. Cell Adh Migr. 2008;2:243-5.

Hynes RO. Integrins: bidirectional, allosteric signaling machines. Cell. 2002;110:673-87.

Kemmner W, Morgenthaler J, Brossmer R. Alterations in cell surface carbohydrate composition of a human colon carcinoma cell line affect adhesion to extracellular matrix components. Biochimie. 1992;74:117-22.

Kremser ME, Przybylo M, Hoja-Lukowicz D, Pochec E, Amoresano A, Carpentieri A, et al. Characterisation of alpha3beta1 and alpha(v)beta3 integrin N-oligosaccharides in metastatic melanoma WM9 and WM239 cell lines. Biochim Biophys Acta. 2008;1780:1421-31.

Lafrenie RM, Podor TJ, Buchanan MR, Orr FW. Up-regulated biosynthesis and expression of endothelial cell vitronectin receptor enhances cancer cell adhesion. Cancer Res. 1992;52:2202-8.

Lawrence MB, Springer TA. Leukocytes roll on a selectin at physiologic flow rates: distinction from and prerequisite for adhesion through integrins. Cell. 1991;65:859-73.

Lee M, Lee HJ, Seo WD, Park KH, Lee YS. Sialylation of integrin beta1 is involved in radiationinduced adhesion and migration in human colon cancer cells. Int J Radiat Oncol Biol Phys. 2010;76:1528-36. 
Lee $\mathrm{SH}$, Cheng H, Yuan Y, Wu S. Regulation of ionizing radiation-induced adhesion of breast cancer cells to fibronectin by alpha5beta1 integrin. Radiat Res. 2014;181:650-8.

Lin S, Kemmner W, Grigull S, Schlag PM. Cell surface alpha 2,6 sialylation affects adhesion of breast carcinoma cells. Exp Cell Res. 2002;276:101-10.

Pretzlaff RK, Xue WW, Rowin ME. Sialidase treatment exposes the beta1-integrin active ligand binding site on HL60 cells and increases binding to fibronectin. Cell Adhes Commun. 2000;7:491-500.

Rae JM, Creighton CJ, Meck JM, Haddad BR, Johnson MD. MDA-MB-435 cells are derived from M14 melanoma cells--a loss for breast cancer, but a boon for melanoma research. Breast Cancer Res Treat. 2007;104:13-9.

Reddy BV, Kalraiya RD. Sialilated beta1,6 branched N-oligosaccharides modulate adhesion, chemotaxis and motility of melanoma cells: Effect on invasion and spontaneous metastasis properties. Biochim Biophys Acta. 2006;1760:1393-402.

Saito M, Sugano K, Nagai Y. Action of Arthrobacter ureafaciens sialidase on sialoglycolipid substrates. Mode of action and highly specific recognition of the oligosaccharide moiety of ganglioside GM1. J Biol Chem. 1979;254:7845-54.

Seales EC, Jurado GA, Brunson BA, Wakefield JK, Frost AR, Bellis SL. Hypersialylation of beta1 integrins, observed in colon adenocarcinoma, may contribute to cancer progression by up-regulating cell motility. Cancer Res. 2005a;65:4645-52.

Seales EC, Jurado GA, Singhal A, Bellis SL. Ras oncogene directs expression of a differentially sialylated, functionally altered beta1 integrin. Oncogene. 2003;22:7137-45.

Seales EC, Shaikh FM, Woodard-Grice AV, Aggarwal P, McBrayer AC, Hennessy KM, et al. A protein kinase C/Ras/ERK signaling pathway activates myeloid fibronectin receptors by altering beta1 integrin sialylation. J Biol Chem. 2005b;280:37610-5.

Semel AC, Seales EC, Singhal A, Eklund EA, Colley KJ, Bellis SL. Hyposialylation of integrins stimulates the activity of myeloid fibronectin receptors. J Biol Chem. 2002;277:32830-6.

Shaikh FM, Seales EC, Clem WC, Hennessy KM, Zhuo Y, Bellis SL. Tumor cell migration and invasion are regulated by expression of variant integrin glycoforms. Exp Cell Res. 2008;314:2941-50.

Shibuya N, Goldstein IJ, Broekaert WF, Nsimba-Lubaki M, Peeters B, Peumans WJ. The elderberry (Sambucus nigra L.) bark lectin recognizes the Neu5Ac(alpha 2-6)Gal/GalNAc sequence. J Biol Chem. 1987;262:1596-601.

St Geme JW, 3rd. The HMW1 adhesin of nontypeable Haemophilus influenzae recognizes sialylated glycoprotein receptors on cultured human epithelial cells. Infect Immun. 1994;62:3881-9.

Taatjes DJ, Roth J, Peumans W, Goldstein IJ. Elderberry bark lectin--gold techniques for the detection of Neu5Ac (alpha 2,6) Gal/GalNAc sequences: applications and limitations. Histochem J. 1988;20:478-90.

Uemura T, Shiozaki K, Yamaguchi K, Miyazaki S, Satomi S, Kato K, et al. Contribution of sialidase NEU1 to suppression of metastasis of human colon cancer cells through desialylation of integrin beta4. Oncogene. 2009;28:1218-29.

Vercoutter-Edouart AS, Slomianny MC, Dekeyzer-Beseme O, Haeuw JF, Michalski JC. Glycoproteomics and glycomics investigation of membrane N-glycosylproteins from human colon carcinoma cells. Proteomics. 2008;8:3236-56.

Yamamoto H, Kaneko Y, Rebbaa A, Bremer EG, Moskal JR. alpha2,6-Sialyltransferase gene transfection into a human glioma cell line (U373 MG) results in decreased invasivity. J Neurochem. 1997;68:2566-76.

Yamamoto H, Oviedo A, Sweeley C, Saito T, Moskal JR. Alpha2,6-sialylation of cell-surface Nglycans inhibits glioma formation in vivo. Cancer Res. 2001;61:6822-9. 
2 Fig. 1. Sialylation of relevant integrins on MDA-MB-231 and MCF-7 cells. For lectin

3 affinity assay, $300 \mu \mathrm{g}$ cell lysate of MDA-MB-231 and MCF-7 cells were harvested and

4 incubated with $50 \mu \mathrm{g} / \mathrm{ml}$ biotinylated SNA at $4^{\circ} \mathrm{C}$ overnight. Streptavidin agarose beads

5 were then added for additional 4 hours incubation at $4^{\circ} \mathrm{C}$. Beads were washed and

6 boiled before loading to the gel. Loading control for lectin affinity assay was collected

7 before adding biotinylated SNA. For western blotting, $100 \mu \mathrm{g}$ cell lysate was loaded.

8 This figure is representative of 3 independent experiments.

10 Fig. 2. Effect of sialidase on adhesion of MDA-MB-231 cells, MCF-7 cells and HT-29 11 cells to ECM. (A) MDA-MB-231 cells, (B) MCF-7 cells and (C) HT-29 cells were 12 harvested and treated with $0.1 \mathrm{U} / \mathrm{ml}$ V. cholera (VC), C. perfringens (CP), and $A$. 13 ureafaciens (AU) sialidase in DPBS for $30 \mathrm{~min}$ at $37^{\circ} \mathrm{C}$. Cells were seeded into each 14 well of fibronectin (FN) or collagen IV (Col IV) pre-coated strips. Cells were washed and 15 stained after $30 \mathrm{~min}$ incubation at $37^{\circ} \mathrm{C}$. Absorbance was normalized to the control cells 16 incubated in DPBS without sialidase. Duplicate samples were prepared in each 17 experiment. Data shown are the means $\pm S D, n=5 . *: p<0.05$ versus untreated control 18 group.

20 Fig. 3. Detection of sialic acid cleavage on MDA-MB-231 and MCF-7 cells by flow 21 cytometry after sialidase treatment. (A) MDA-MB-231 and (B) MCF-7 cells were 22 harvested and treated with $0.1 \mathrm{U} / \mathrm{ml}$ V. cholera (VC), C. perfringens (CP), or $A$. 
1 ureafaciens $(\mathrm{AU})$ sialidase in DPBS for $30 \mathrm{~min}$ at $37^{\circ} \mathrm{C}$. SNA binding, CD15 expression

2 and CD15s expression on MDA-MB-231 and MCF-7 cells were measured by flow

3 cytometric analysis. Red dotted lines represent background (secondary antibody alone),

4 blue lines represent control cells incubated in DPBS without sialidase and orange lines

5 represent sialidase treated cells. Quantification of flow cytometry by normalizing mean

6 fluorescence intensity relative to control cells is shown in C and D. These figures are

7 representative of 3 independent experiments.

9 Fig. 4. Surface expression of relevant integrins on MDA-MB-231 and MCF-7 cells after 10 sialidase treatment. (A) MDA-MB-231 cells and (B) MCF-7 cells were harvested and 11 treated with $0.1 \mathrm{U} / \mathrm{ml} V$. cholerae, C. perfringens, and A. ureafaciens sialidase in DPBS

12 for $30 \mathrm{~min}$ at $37^{\circ} \mathrm{C}$. Expression of $\alpha 5, \beta 1$ and activated $\beta 1$ integrin on MDA-MB-231 13 cells, and $\beta 1$ integrin and activated $\beta 1$ integrin on MCF-7 cells were analyzed by flow 14 cytometry. Red dotted lines represent isotype control, blue lines represent control cells 15 incubated in DPBS without sialidase treatment and orange lines represent sialidase 16 treated cells. These figures are representative of 3 independent experiments.

Fig. 5. Effect of sialidase on migration and invasion of MDA-MB-231 cells. (A) MDA-MB-

19231 cells were grown on a fibronectin-coated plate to reach confluence and were 20 treated with $0.1 \mathrm{U} / \mathrm{ml}$ C. perfringens $(\mathrm{CP})$, and $\mathrm{A}$. ureafaciens $(\mathrm{AU})$ sialidase in DPBS for

$2130 \mathrm{~min}$ at $37^{\circ} \mathrm{C}$. The wound was then made using a $200 \mu \mathrm{l}$ pipette tip. Cells were 22 incubated in FBS free medium for $7 \mathrm{~h}$ at $37^{\circ} \mathrm{C}$. Photographs were taken at $0 \mathrm{~h}$ and $7 \mathrm{~h}$. 
1 Gap distances were shown as the ratio of $7 \mathrm{~h}$ to $0 \mathrm{~h}$. Duplicate samples were prepared

2 in each experiment. Data shown are the means $\pm S D, n=4 . *$ : $p<0.05$ versus control

3 group. Scale bar, $250 \mu \mathrm{m}$. (B) MDA-MB-231 cells were harvested and treated with

4 sialidases as described in (A). $1 \times 10^{5}$ MDA-MB-231 cells were seeded into inner

5 chamber of transwell inserts coated with fibronectin (FN). After $24 \mathrm{~h}$ incubation at $37^{\circ} \mathrm{C}$,

6 cells migrated to the lower surface were fixed, stained and then counted under a

7 microscope from 4 different fields. Duplicate samples were prepared in each

8 experiment. Data shown are the means $\pm S D, n=3 . \quad *$ : $p<0.05$ versus control group.

9 Scale bar, $50 \mu \mathrm{m}$. 


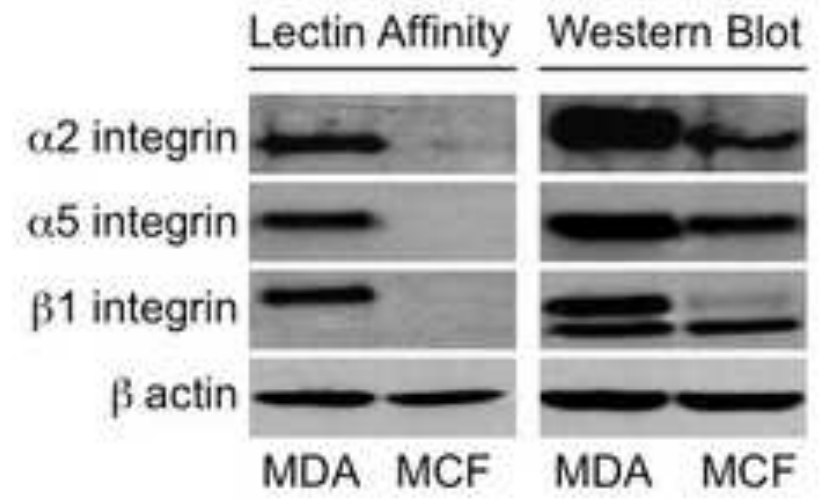




\section{A. MDA-MB-231 Cells}

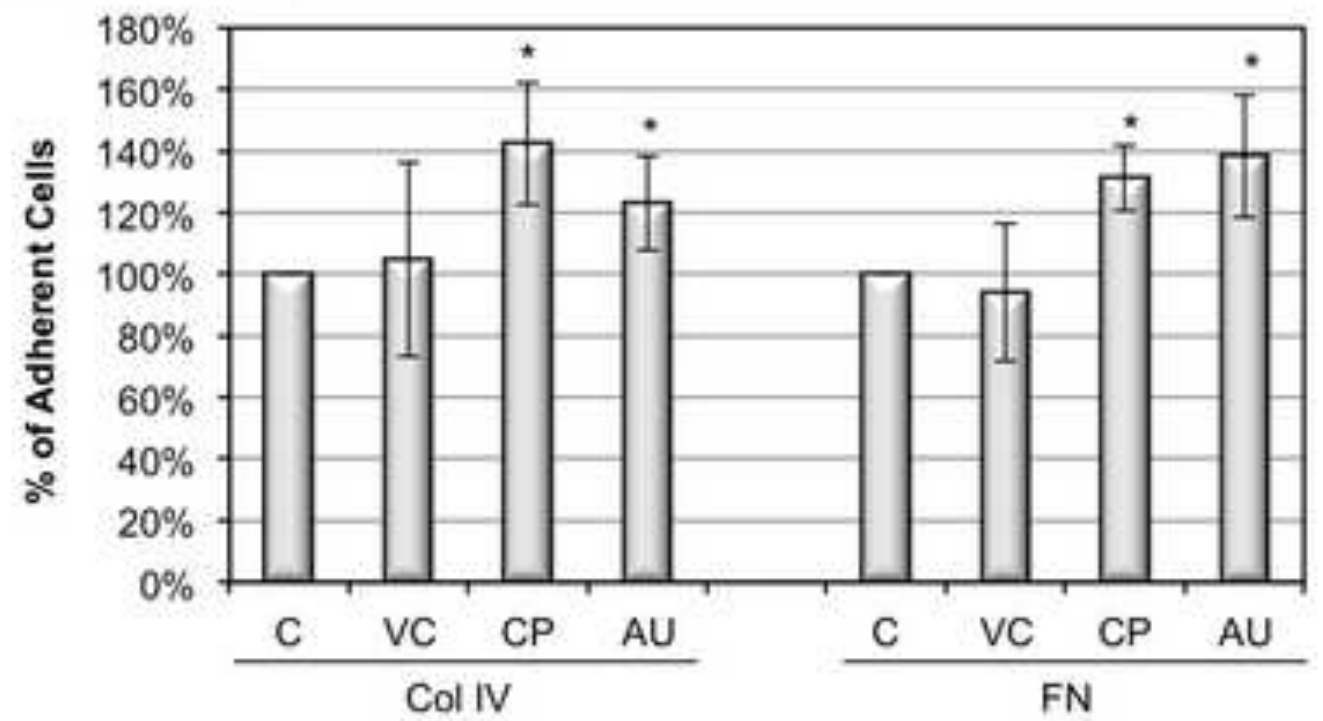

B. MCF-7 Cells

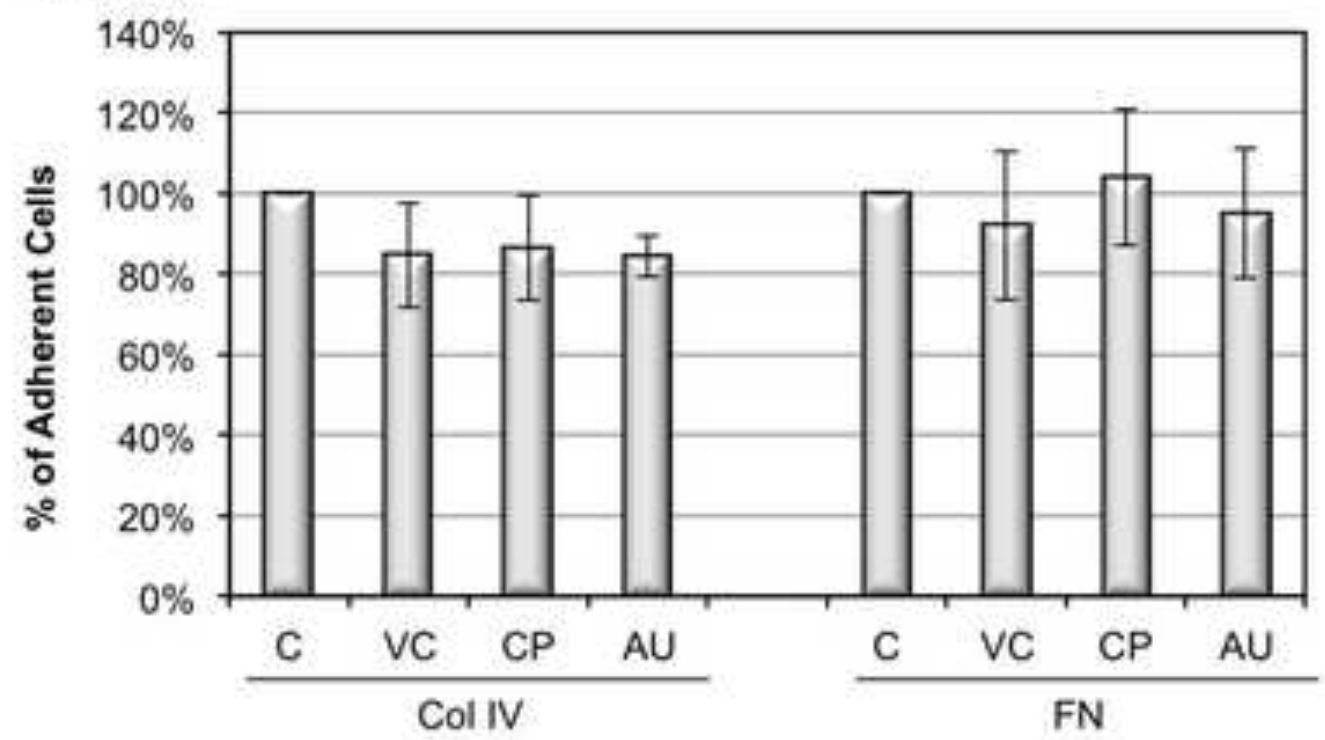

C. HT-29 Cells

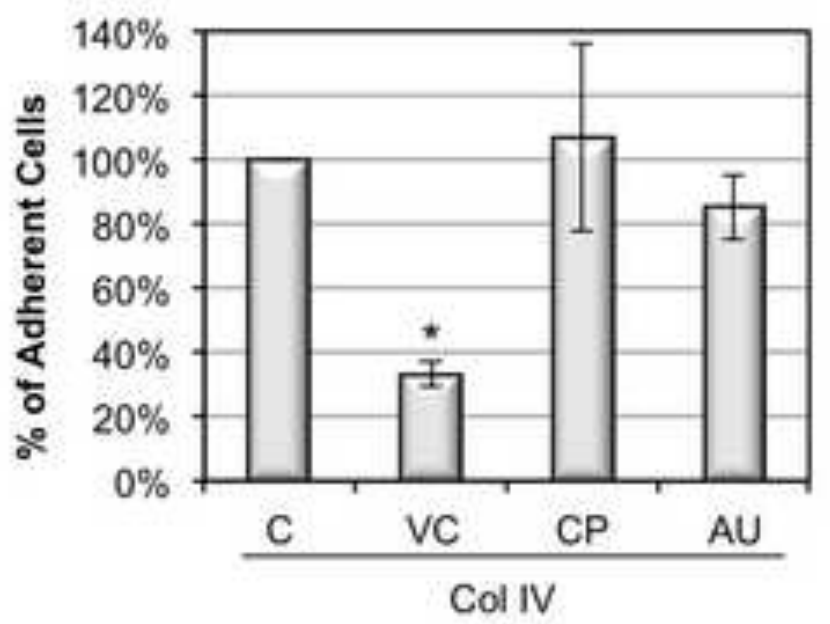




\section{A. MDA-MB-231}

V. cholerae

C. perfringens A. ureafaciens
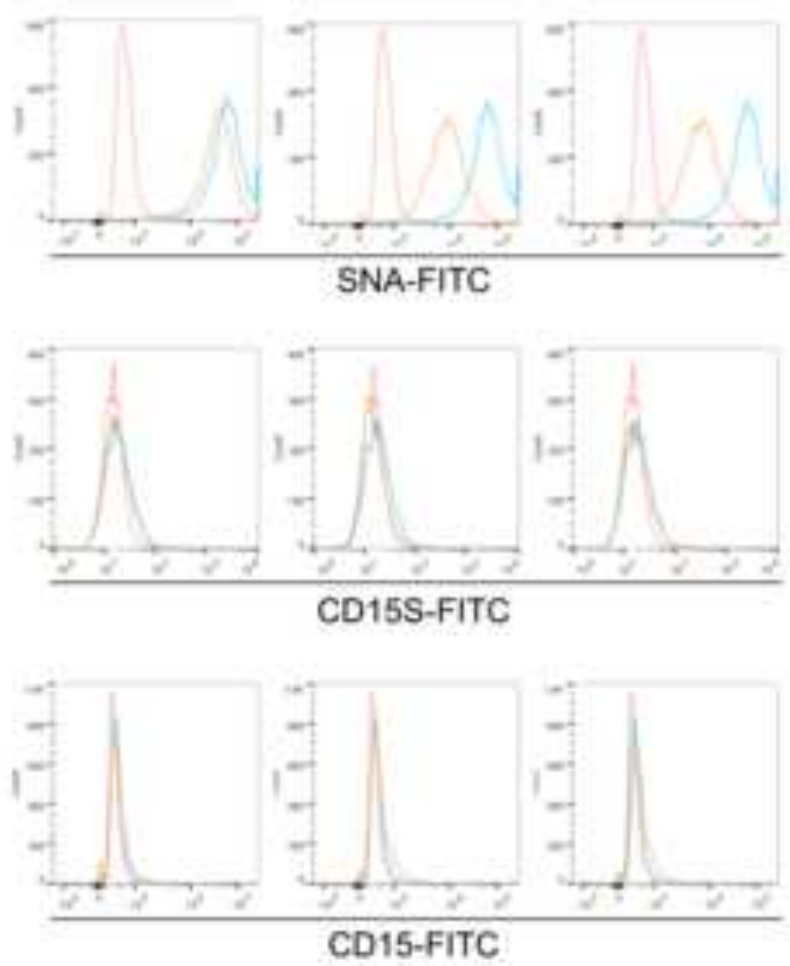

\section{MDA-MB-231}

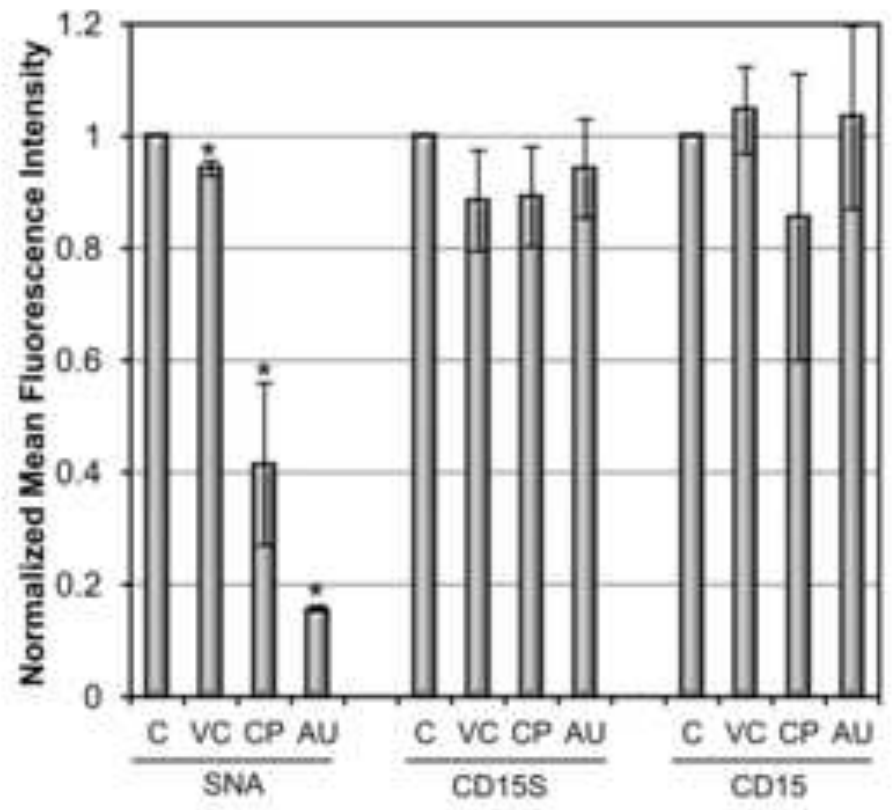

B. MCF-7
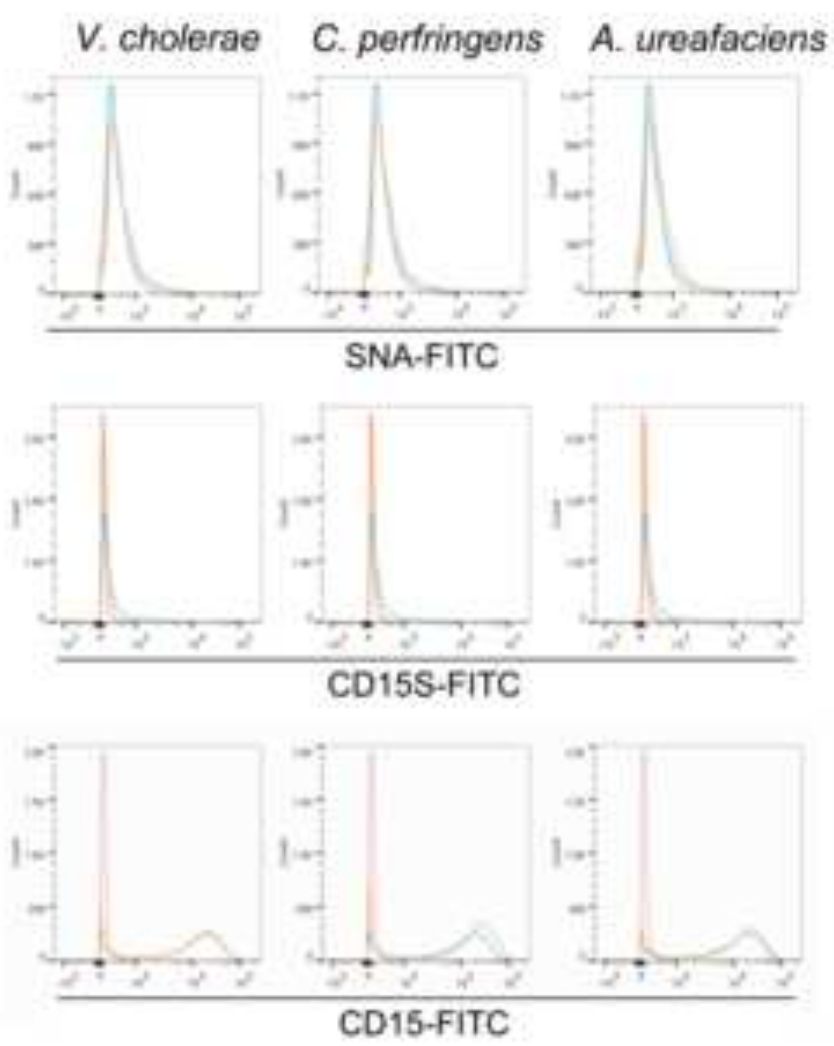

D. MCF-7

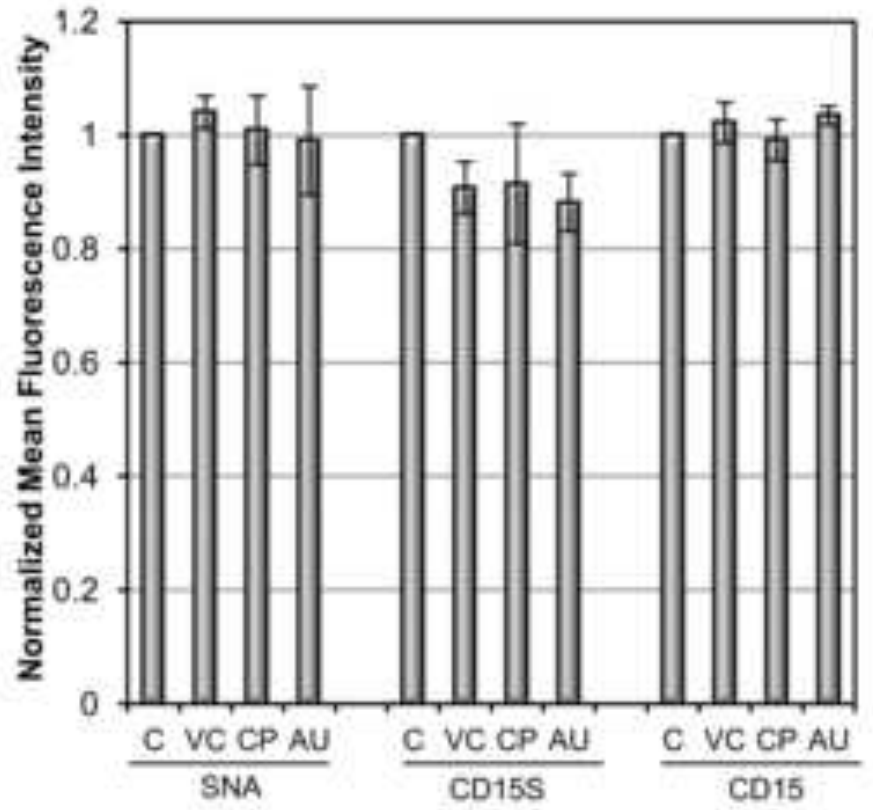




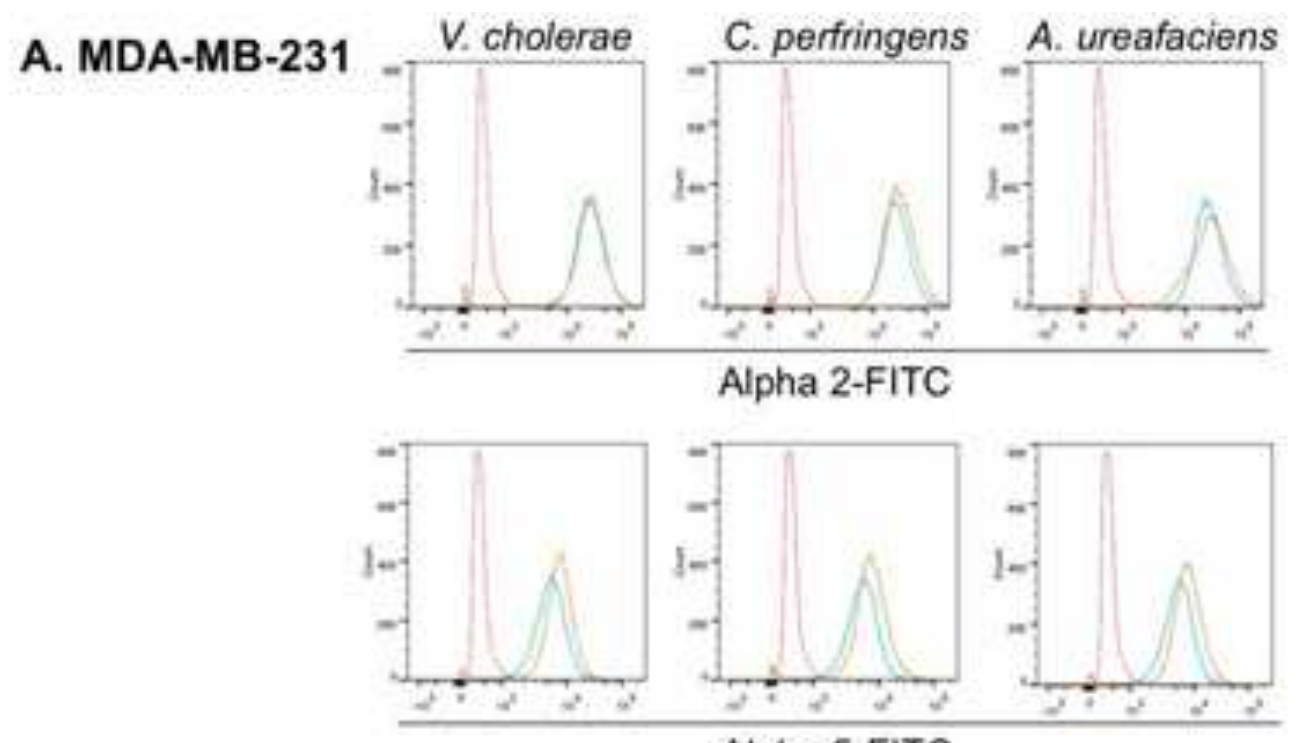

Alpha 5-FITC
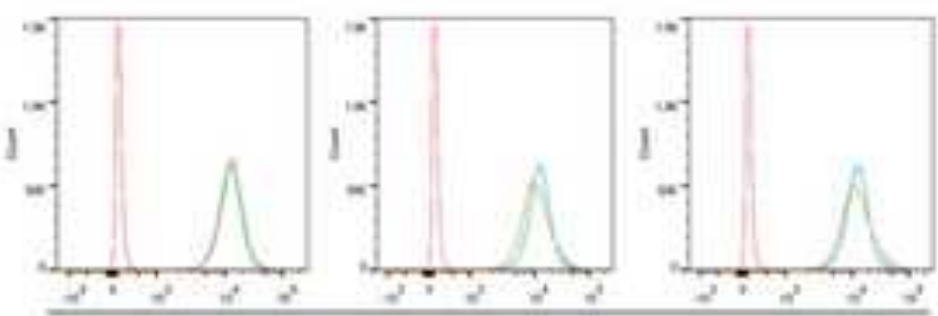

Beta 1-FITC

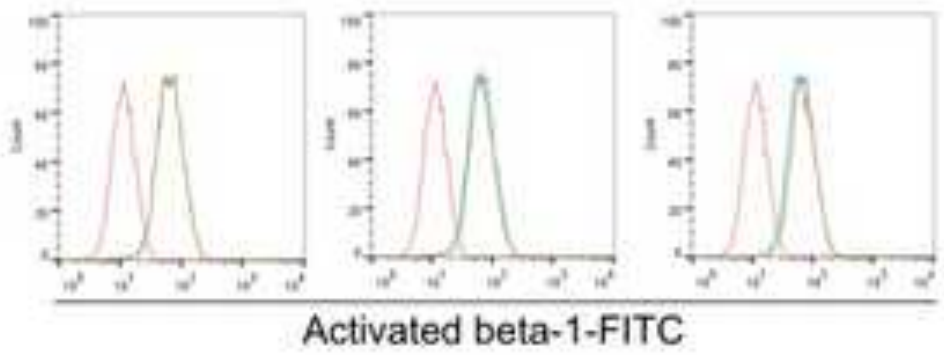

\section{B. MCF-7}
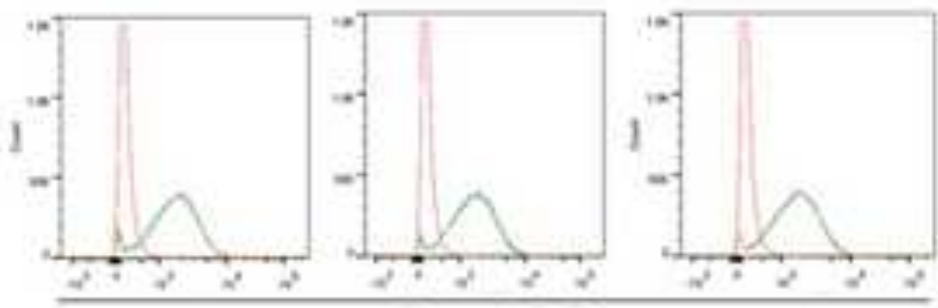

\section{Beta 1-FITC}
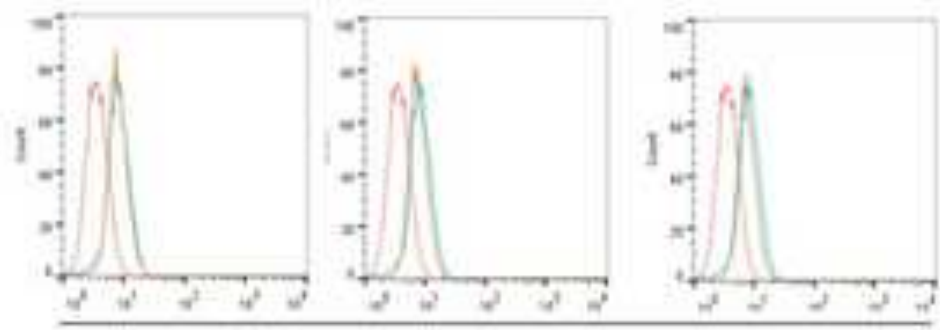

Activated beta-1-FITC 
A.
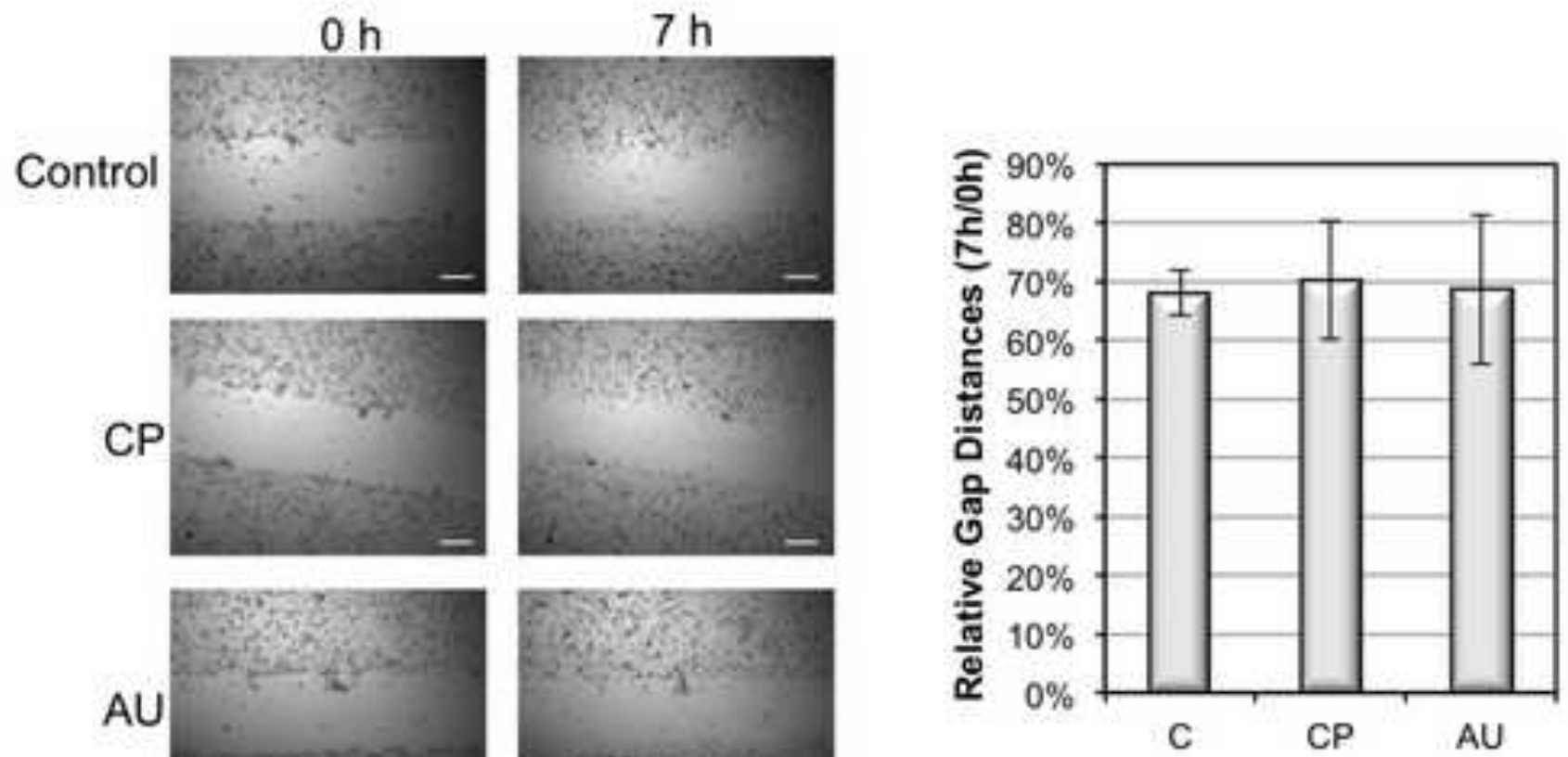

B.
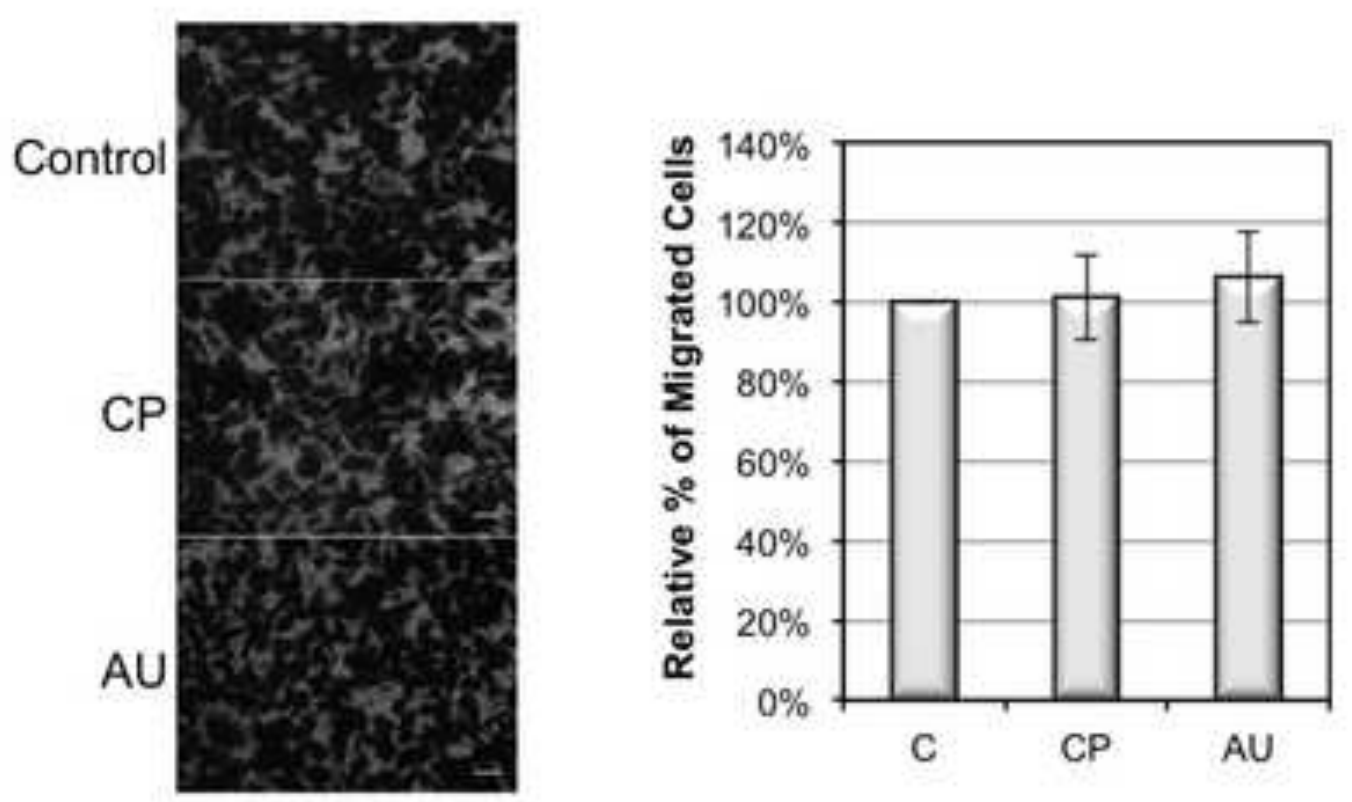